1 Faculdade de Medicina de Marília (Famema), Programa de Residência Integrada Multiprofissional em Saúde Coletiva Marília (SP), Brasil. danielsalvador.psico@gmail. com

2 Faculdade de Medicina de Marília (Famema),

Programa de Residência Integrada Multiprofissional em Saúde Coletiva Marília (SP), Brasil. Universidade Estadual Paulista Júlio de Mesquita Filho (Unesp), Faculdade de Medicina, Programa de Pós-Graduação em Saúde Coletiva - Botucatu (SP), Brasil.

danimassihpio@hotmail.com

\section{Apoio Matricial e Capsi: desafios do cenário na implantação do matriciamento em saúde mental}

\author{
Matrix Support and Capsi: scenario challenges in the deployment of \\ the mental health matrix
}

Daniel Barboza Salvador', Danielle Abdel Massih Pio ${ }^{2}$

RESUMO Este estudo qualitativo teve como objetivo explorar o conhecimento e a experiência de uma equipe de Centro de Atenção Psicossocial Infantil (Capsi) acerca da estratégia do Apoio Matricial (AM), com a participação de cinco profissionais da equipe de referência e quatro residentes multiprofissionais. A análise utilizou-se de dois encontros de Grupo Focal no Capsi em 2015-2016 e do Discurso do Sujeito Coletivo. Como resultado, não se chegou a uma definição clara sobre o tema e não se reconheceu uma experiência com AM, tendo dificuldades para implantar esta estratégia dentro das ações de cuidado em saúde mental. Levanta-se a necessidade de articulação de uma rede que realmente consiga contribuir com a mudança de paradigma do modelo médico hegemônico.

PALAVRAS-CHAVE Saúde pública. Serviços de saúde mental. Pesquisa qualitativa.

ABSTRACT This qualitative study aimed to explore the knowledge and experience of a Center for Child Psychosocial Care (Centro de Atenção Psicossocial Infantil - Capsi) team about Matrix Support Strategy (AM) by means of the participation of five professionals' reference team and four multidisciplinary residents. We used two Focus Groups in Capsi premises during 2015-2016 and applied the Collective Subject Discourse to analyze the speeches. As a result, it was not found any clear definition of the subject and no AM experience was recognized, struggling the implementation of the strategy within care actions in mental health. We conclude by the need to articulate a network that can really contribute to the paradigm shift of the hegemonic medical model.

KEYWORDS Public health. Mental health services. Qualitative research. 


\section{Introdução}

O Centro de Atenção Psicossocial Infantil (Capsi) é um serviço de saúde que atende crianças e adolescentes com transtorno mental grave persistente e que fazem uso de crack, álcool ou outras drogas. Ainda, tem a responsabilidade de ser o ordenador da rede de cuidados em saúde mental infanto-juvenil e de fazer valer as diretrizes das políticas públicas de saúde mental no âmbito do seu território (BRASIL, 2005).

Seguindo-se a proposta da Organização Mundial da Saúde e da Federação Mundial de Saúde Mental, priorizou-se a construção de uma Política de Saúde Mental voltada para a infância e adolescência cujo princípio é a ideia de que a criança ou o adolescente é um sujeito (BRASIL, 2005). Portanto, é um sujeito que tem direitos, é singular e que, por ter voz própria, precisa ser escutado. Como ainda é um sujeito em formação, o cuidado em saúde está associado à construção da subjetividade e à constituição da saúde mental (BOWLBY, 2002).

Por isso, a equipe da atenção básica pode ser essencial na minimização, resolução e promoção da saúde mental da criança logo no início da sua evolução, pois pode colocar o núcleo familiar como referência para compreender os problemas de saúde mental, visto que as relações primárias são fundamentais na formação do sujeito (RANÑA, 2010).

No entanto, a saúde mental enfrenta muitos desafios. A efetivação dessa política necessita de ações que não considerem apenas a legitimação dos princípios do Sistema Único de Saúde (SUS) e da Reforma Psiquiátrica, mas também as características específicas dessa população em termos subjetivos e sociais (BRASIL, 2005).

Diante desse contexto, os profissionais que trabalham na atenção básica precisam do suporte de uma equipe especializada. Assim, tem-se o Apoio Matricial (AM) e as Equipes de Referência (ER) como modelos que possibilitam a reorganização do trabalho e se configuram como uma metodologia para a gestão em saúde. Espera-se com essa metodologia proporcionar uma integração dialógica entre as diferentes especialidades e profissões e a corresponsabilização. Ainda, o AM assegura uma retaguarda assistencial e um suporte técnico pedagógico às ER, visando avançar na construção do modelo que tem a atenção básica como cuidador principal (BRASIL, 2011).

Assim, o AM, segundo Campos (1999), tem como meta a propagação da clínica ampliada nas equipes interdisciplinares de saúde, proporcionando um tipo de cuidado colaborativo na atenção básica. Desvia a lógica dos encaminhamentos indiscriminados para uma lógica da corresponsabilização, fortalecendo a produção de saúde a partir da resolutividade da assistência em saúde.

Ao concordar com Campos (1999), Chiaverini (2011) entende que AM é um novo modelo de produzir saúde em que duas ou mais equipes, num processo de construção compartilhada, criam uma proposta de intervenção pedagógico-terapêutica. Ainda, não se define como supervisão, mas como uma transformação no modelo de conduta dos casos, causando uma transformação no modelo tradicional de transferência dos casos para outra equipe de referência, ou seja, a tradicional lógica de referência e contrarreferência presente no modelo médico hegemônico.

Essa lógica, que utiliza as guias de referência e contrarreferência, favorece a falta de responsabilização e alienação dos profissionais em relação ao seu objetivo, que é a produção de saúde (FIGUEIREDO, 2006). Por isso, tem-se como substituto a lógica do encaminhamento implicado (BRASIL, 2005), que valoriza o encontro entre profissionais, a continuidade e a integralidade do cuidado, sendo que o AM é uma metodologia potente para a mudança desses paradigmas.

Considerando a importância do AM, esta pesquisa teve como objetivo explorar o conhecimento e a experiência de uma equipe 
de saúde mental acerca da estratégia do matriciamento. $\mathrm{O}$ estudo foi realizado em um município do estado de São Paulo, no qual os pesquisadores tinham acesso enquanto profissionais da saúde, por meio de atividades da residência multiprofissional e de supervisão docente. Esse município possui dois Centros de Atenção Psicossocial (Caps), um Caps adulto (Caps II) e um Capsi, vinculados diretamente à Secretaria Municipal de Saúde. Decidiu-se não incluir o serviço do Caps II na pesquisa devido à participação ativa dos pesquisadores na tentativa recente de incluir o AM na agenda da equipe, fato que poderia influenciar os resultados da pesquisa.

A tentativa de realização do AM em outro cenário de Caps, nesse mesmo município, foi um disparador para a construção da hipótese de que a micropolítica do cotidiano institucional cristaliza significados que funcionam como defesa contra o desconhecido, o que dificulta a construção do novo. Diante dos desafios de se realizar AM em uma instituição de saúde mental, visto que existiram dúvidas desde a definição desse tema entre os profissionais, o objetivo foi o de encontrar repostas para as dificuldades de se inserir tal estratégia no planejamento e nas ações de cuidado.

\section{Métodos}

Trata-se de um estudo do tipo qualitativo e exploratório descritivo, que utilizou a técnica do Grupo Focal (GF) para a coleta de dados. Os participantes foram selecionados de forma intencional. A equipe do Capsi, no momento da pesquisa, tinha um enfermeiro, um médico psiquiatra, um psicopedagogo, um assistente social e quatro técnicos ou auxiliares de enfermagem, totalizando oito profissionais. No caso, a equipe mínima tem quatro profissionais de nível superior, sem considerar a categoria médica, e cinco profissionais de nível médio. A condição que dificultou a realização da pesquisa foi o número reduzido de profissionais da equipe fixa. Assim, férias, reuniões com a gestão e afastamentos foram condições que impossibilitaram a participação de alguns profissionais, resultando em uma participação incompleta. No total, participaram nove profissionais, sendo cinco pertencentes à equipe de referência do Capsi e quatro residentes multiprofissionais em saúde mental, que atuavam em tempo integral na unidade há aproximadamente um ano: uma psicóloga, uma enfermeira, uma terapeuta ocupacional e uma assistente social. Os participantes serão identificados pela letra $\mathrm{P}$ seguida de um número ( $\mathrm{P} 1, \mathrm{P} 2$ e assim sucessivamente).

Para a coleta de dados, foram realizados dois encontros de GF entre os meses de dezembro de 2015 e fevereiro de 2016. A duração média dos encontros foi de 45 minutos. É possível pensar o GF como uma técnica de entrevista em grupo, na qual a interação entre os participantes se configura como parte do método, com ampla problematização acerca de um tema ou foco específico. Nesse sentido, o que se pretende é analisar a interação entre os participantes e a forma como os significados são construídos em grupo (BACKES ET AL., 2011).

As sessões ocorreram na sala de reunião do Capsi, foram gravadas em áudio, transcritas, analisadas e apresentadas aos mesmos participantes com o intuito de validarem a transcrição. Um dos pesquisadores mediou o grupo e ocupou o lugar de moderador, enquanto outro profissional, convidado pelos pesquisadores e não pertencente à equipe, ocupou na função de observador.

Para introduzir o assunto e disparar as discussões, foi elaborado um material de estímulo que trouxe a temática 'Matriciamento nos serviços de saúde ou saúde mental', com a aplicação do roteiro de entrevista semiestruturado para guiar a discussão sobre as concepções e experiências de tal estratégia no serviço. O GF foi gravado em áudio e transcrito para a análise dos dados.

Para análise dos dados, foram utilizados os 
fundamentos da clínica ampliada, da Política Nacional de Humanização e da Reforma Psiquiátrica, relacionando-os com as teorias da psicanálise e com conceitos de análise institucional e da psicodinâmica do trabalho.

Como referencial metodológico, utilizou-se o Discurso do Sujeito Coletivo (DSC) (LEFÉVRE; LEFÉVRE; MARQUES, 2009). A técnica do DSC consiste em reunir conteúdos de depoimentos individuais com sentidos semelhantes, construindo discursos únicos redigidos na primeira pessoa do singular, de modo a produzir um efeito de 'coletividade falando'.

Desse modo, tentou-se identificar as Ideias Centrais (IC) presentes nos discursos dos participantes e as Expressões Chave (ECH) que as representam com o objetivo de construir discursos únicos redigidos na primeira pessoa do singular, os quais ilustram os pensamentos, atitudes, concepções e práticas acerca do tema proposto (ANDRADE; DUARTE; MAMEDE, 2009).
As intenções do pesquisador e os objetivos da pesquisa foram esclarecidos previamente à equipe. $\mathrm{O}$ estudo foi aprovado pelo Comitê de Ética em Pesquisa (CEP), com o parecer de número 1.347.158.

\section{Resultados e discussão}

A partir das transcrições do GF, identificaram-se as IC extraídas do discurso dos participantes e suas representações em ECH, com as quais foram construídos os DSCs. Após a construção destes, identificaram-se seis temáticas, a partir das quais organizaram-se quadros com os discursos que as representavam e realizaram-se as discussões de acordo com os temas e discursos da equipe do Capsi. Apresentam-se a seguir os discursos de cada temática com as respectivas discussões.

\section{Conhecimento e o conceito sobre o AM}

Quadro 1. IC* e DSC** sobre conceito de $\mathrm{AM}^{\star \star \star}$

\begin{tabular}{ll}
\hline & Dúvidas referentes ao conceito de AM \\
IC 1 (DSC) - "E o que seria o AM, num termo mais popular?" (P3). & (DSC) - "Tentamos fazer matriciamento, mas, devido às dificuldades, também fiquei na dúvida quanto à defini- \\
& cão mesmo" (E9). \\
\hline IC 2 & Dificuldade para compreender o conceito devido à pouca experiência \\
& (DSC) - "Não é um conceito fácil de compreender, e quando não está no exercício, fica ainda mais difícil" (P2). \\
\hline
\end{tabular}

${ }^{\star} \mid \mathrm{C}$ - Ideia Central / ${ }^{* \star}$ DSC - Discurso do Sujeito Coletivo / ${ }^{* \star \star} \mathrm{AM}$ - Apoio Matricial.

Em outras pesquisas (ELIZAETE, 2009; GARCIA JÚNIOR; ÁVILA-NASCIMENTO, 2012; PRESTES, 2011), concluiu-se que as equipes não possuíam uma definição clara sobre AM, apresentando dúvidas referentes ao conceito e dificuldades para empregar a metodologia. Assim, não foi diferente com a equipe que participou desta pesquisa. Isso pode ser confirmado a partir da resposta: "E o que seria o $A M$, num termo mais popular?" (P3). Nota-se que a definição de AM não foi clara e poderia até ser considerada desconhecida, se analisada apenas essa resposta. No entanto, a generalização seria imprudente neste momento, ao considerar apenas o elemento conhecimento.

Para essa equipe, o conceito de AM não era de fácil compreensão e teria uma definição complexa e teoricamente bem 
fundamentada. Não estavam completamente no caminho errado, tendo em vista que o AM tem definição teórica e fundamentação próprias (CAMPOS, 1999; CAMPOS; DOMITTI, 2007; CHIAVERINI, 2011). No entanto, espera-se que cada equipe, em determinadas condições e considerando a singularidade de sua rede de saúde, construa os seus conceitos a partir da prática e da leitura de material teórico. Assim, como propõe Merhy (2013), é necessário engravidar as palavras, de forma que os conceitos pré-estabelecidos ganhem significados particulares que contenham na sua definição as palavras dos profissionais, adquiridas a partir das experiências em saúde, que teve o mundo do trabalho como escola, compartilhando conhecimentos e experimentações criativas para superar os desafios e atender integralmente às necessidades de saúde, produzindo um cuidado em saúde singular.

\section{Experiência com AM}

Quadro 2. IC* e DSC** sobre a experiência com $\mathrm{AM}^{\star \star \star}$

\begin{tabular}{ll}
\hline & A equipe considerou que não teve uma experiência com AM \\
IC 3 & (DSC) - "Eu acho que a gente nunca viveu um AM" (P1). \\
(DSC) - "Entendo que é uma forma de mudar a questão da referência e contrarreferência a partir da discussão \\
de casos, mas como podemos fazer isso?" (E2). \\
A equipe não sabia como fazer AM \\
(DSC) - "A gente pega um pouquinho aqui, outro porquinho ali, em um lugar matricial de uma forma, em outro \\
IC 4 lugar de outra forma. Pegamos isso de outras reuniões que participamos, fora que eles falam que estão fazendo \\
matriciamento... conseguia ter uma ideia geral do que se tratava, mas não sabia como fazer" (P4).
\end{tabular}

${ }^{*} \mathrm{IC}$ - Ideia Central / ${ }^{* \star}$ DSC - Discurso do Sujeito Coletivo / ${ }^{\star \star \star} \mathrm{AM}$ - Apoio Matricial.

A experiência em saúde é fundamental para a construção de novos significados e formação de conceitos, conforme Merhy (2013) defendeu. Entretanto, a equipe entrevistada considerou que nunca tiveram uma experiência de AM, onde ocuparam o lugar de protagonistas da criação de espaços de diálogo e produção de novo arranjo organizacional que garantisse um cuidado integral e continuado. Por não terem vivenciado um matriciamento, algumas dúvidas foram geradas e, por isso, os conhecimentos sobre o tema sofreram distorções, incompreensões e insegurança. Portanto, não se tornou possível a construção de novos significados.
Pelo mesmo motivo, houve dúvidas na relação entre prática e teoria sobre AM. E essas dúvidas retornaram para os conceitos teóricos, pois, no desencontro entre a pouca vivência e o estudo teórico, colocaram em questão a produção de conhecimento, visto que podia ocorrer um confronto entre idealização e realidade.

Reconhecendo que o conceito de AM não estava claro e bem definido para a equipe e diante da situação de pouca vivência com a metodologia, a pesquisa tentou identificar outras experiências de aproximação com esse tema. Ou seja, a pesquisa visou a identificar se a equipe escutou uma experiência de outra equipe, se observaram um trabalho 
realizado ou se leram sobre uma experiência na literatura.

\section{Percepção sobre AM}

Quadro 3. IC* e DSC*^ sobre a percepção de $A M^{\star \star \star}$

\begin{tabular}{ll}
\hline IC 5 Percepção que o AM está na dimensão da macropolítica \\
(DSC) - "Quando penso em AM, penso em algo macro, algo muito grande" (P1). \\
(DSC) - "E quando fala de matriciamento como algo grande, uma coisa maior, parece que os pequenos não \\
estão se ajudando" (E5). \\
Percepção que o AM se configura como uma tecnologia de gestão que modifica a comunicação dentro da \\
rede de atenção à saúde \\
(DSC) - "Eu sei que, pensando no que eu já vi, é uma contenção de uma tecnologia de gestão. Eu acho que tem \\
essa proposta de mudança da comunicação entre os serviços" (P1). \\
Percepção que o AM não é um favor \\
(DSC) - "Tem que ter esse olhar para saber o que é apoio e o que é favor. Parece que todo mundo faz favor e não \\
tem uma coisa combinada. Às vezes tem dificuldade, porque tudo parece que é favor. Tem uma diferença entre \\
apoio e fazer um favor" (P5). \\
IC 7 Dúvidas quanto à responsabilidade do apoio matricial \\
(DSC) - "Buscando a literatura, sabemos que o Caps é uma equipe que pode matriciar. Mas, pensando de manei- \\
ra mais abrangente, eu tenho dúvidas. Deveria ser a equipe do Caps para matriciar ou ter uma equipe específica \\
para fazer esse apoio matricial, que ficaria mais próxima das equipes que recebem apoio no território?" (P7).
\end{tabular}

${ }^{\star} \mathrm{IC}$ - Ideia Central / ${ }^{\star \star}$ DSC - Discurso do Sujeito Coletivo / ${ }^{* \star *} \mathrm{AM}$ - Apoio Matricial.

Logo, na percepção dessa equipe, o objetivo do AM esteve atrelado a modificar a organização do trabalho em rede e, por isso, seria uma tecnologia de gestão. Tal definição, construída coletivamente, está de acordo com as definições de alguns autores (CAMPOS, 1999; FIGUEIREDO, 2006; CHIAVERINI, 2011), que sugerem o AM como um novo arranjo organizacional do processo de trabalho.

Após algumas conclusões sobre o significado de AM, a equipe pode construir algumas reflexões. Logo, o AM não pode ser compreendido como troca de favores, mas como um compromisso profissional, pois relataram que outras equipes solicitaram apoio no sentido de trocar favores. Esse tipo de relação, segundo a equipe, enfraquecia a produção de trabalho em saúde, pois as relações pareciam depender de interesses.

Em outra reflexão, teve-se a percepção do AM como intervenção complexa e muito ampla, pois parecia ocorrer no nível macro, ficando além da governabilidade da equipe.
No entanto, segundo Cecílio e Matsumoto (2006), para realizar ações de atenção à saúde de forma integral, deve-se enxergar que o 'micro' está no 'macro' e o 'macro' no 'micro', pois a luta pela integralidade, assim como pela equidade, implica necessariamente repensarmos os aspectos do processo de trabalho, gestão, planejamento e construção de novos saberes e práticas em saúde. Logo, o AM ocorre no cotidiano do trabalho da equipe e no encontro com outras equipes, sendo transformadora para si mesmo e para os outros (PRESTES, 2011).

E, nessa construção coletiva de novos conhecimentos, os entrevistados começaram a questionar sobre quem eram os responsáveis em realizar o AM. Sobre isso, não atribuíram tal intervenção a uma profissão específica, mas ao trabalho em equipe. No entanto, reconheciam essa responsabilidade a partir da observação de outras experiências fora do Capsi e por meio da leitura de materiais teóricos, como artigos científicos e cartilhas do 
SUS. Por isso, atribuíram à equipe do Capsi, dentro da Rede de Atenção Psicossocial, responsabilidade acessória a essa intervenção.

Entretanto, por não terem experiência com o AM, a equipe ainda demonstrou muitas dúvidas em relação a essa colocação, pois surgiu entre eles a ideia de que o matriciamento poderia ser realizado por uma equipe formada especificamente para essa intervenção, de forma que atuariam próximas das Equipes de Saúde da Família $(\mathrm{EqSF})$ e a favor do vínculo entre as equipes no território, como as equipes do Núcleo de Apoio à Saúde da Família (Nasf), conforme preconizado pela Política da Atenção Básica a partir da Portaria ${ }^{\circ}{ }^{2488} / 11$. A equipe do Nasf também utiliza o AM como metodologia de gestão, mas não detém sua exclusividade, visto que o matriciamento pode ser utilizado por qualquer equipe de saúde (BRASIL, 2011).

\section{Desafios e viabilidade para realizar o AM}

Quadro 4. IC* e DSC ${ }^{\star \star}$ sobre desafios e a possibilidade para realizar o $\mathrm{AM}^{\star \star \star}$

\begin{tabular}{|c|c|}
\hline IC 9 & $\begin{array}{l}\text { O desafio da corresponsabilização } \\
\text { (DSC) - "O desafio é não ter muito claro o conceito, e os profissionais que têm clara essa definição não consegui- } \\
\text { rem passar para outras equipes. Fica ainda mais difícil quando vamos na atenção básica e as equipes não querem } \\
\text { apoio, querem que a equipe de especialidade realize atendimentos. Eles não querem fazer juntos" (P9). }\end{array}$ \\
\hline IC 10 & $\begin{array}{l}\text { O desafio de articular o trabalho em rede } \\
\text { (DSC) - "Entendo que é uma forma de mudar a questão da referência e contrarreferência a partir da discussão de } \\
\text { casos, mas como podemos fazer isso?" (P3). }\end{array}$ \\
\hline IC 11 & $\begin{array}{l}\text { A equipe de referência reduzida dificulta o matriciamento } \\
\text { (DSC) - "Eu acho que uma dificuldade é formar uma equipe só para o Capsi. Essa é uma dificuldade muito gran- } \\
\text { de que a gente tem. Temos que ter uma equipe apenas para o Capsi" (P1). }\end{array}$ \\
\hline IC 12 & $\begin{array}{l}\text { Desafio de gerar demandas que a equipe de apoio não conseguiria assistir } \\
\text { (DSC) - "O difícil é em relação a mostrar nosso serviço, ofertar um apoio e depois não dar conta da demanda" } \\
\text { (P3). }\end{array}$ \\
\hline IC 13 & $\begin{array}{l}\text { O AM é uma intervenção possível, mas precisa de incentivos para se realizar } \\
\text { (DSC) - "É possível. O problema são as questões trabalhistas ou de gerenciamento. Como o Capsi está com falta } \\
\text { de profissionais, fica mais difícil expor nosso trabalho. Mas, mesmo assim, acredito que é possível dentro da nossa } \\
\text { realidade" (P1). }\end{array}$ \\
\hline
\end{tabular}

Os desafios mostrados por essa equipe do Capsi são comuns aos desafios enfrentados pela política de saúde mental infanto-juvenil. Primeiramente, apesar dos avanços históricos reconhecidos, a criança ainda tem pouca visibilidade no cenário da saúde, incluindo a saúde mental. O cuidado em saúde direcionado à família pode, em muitos casos, ter como objeto o contexto sociocultural dos adultos e não considerar os desejos, a subjetividade e a responsabilidade da criança diante do problema apresentado (CAVALCANTE; JORGE; MOURA-SANTOS, 2012).
Outro desafio é em relação aos profissionais que não possuem formação para atuar com problemas de saúde mental em crianças e adolescentes, o que pode causar um obstáculo à efetivação do cuidado, reduzir a atenção ao tratamento medicamentoso de crianças - que estão em plena formação subjetiva - ou ao encaminhamento ao serviço especializado em razão das dificuldades dos profissionais em reconhecer os problemas de saúde mental infantil. Importante ressaltar que o paradigma associado à loucura também está presente na população infantil, 
mas, nesse caso, provém, de forma especial, dos familiares que não desejam que suas crianças sejam expostas a um diagnóstico psiquiátrico e dos profissionais que não reconhecem os problemas de saúde mental ou não conseguem lidar com esse tipo de problema (TANAKA, 2010).

As questões envolvidas com saúde mental infanto-juvenil atravessam os limites de um caso clínico, pois envolvem também os paradigmas da loucura. Por isso, o cotidiano das equipes de saúde e saúde mental coloca os trabalhadores em contato com o sofrimento, a vulnerabilidade, a desigualdade social, a marginalização, o desamparo, a desassistência, a exclusão social, a dor e até mesmo a morte. Trabalhar com tais aspectos, especialmente com a população infanto-juvenil, demanda um desgaste emocional que, somado à pressão exercida por esse tipo de trabalho, cria um campo propício para estresse e depressão dos trabalhadores.

São contradições encontradas no cotidiano de trabalho que não correspondem às propostas do Ministério da Saúde (MS). A realidade dos trabalhadores evidencia uma sobrecarga de trabalho, pois eles precisam responder às demandas de saúde com um alto nível de desempenho enquanto sua realidade é de sobrecarga de trabalho. Assim, a equipe reduzida é responsável por um território com uma população que está acima do número máximo estabelecido pelo MS. Logo, para cuidar da própria saúde, os trabalhadores passam a limitar seus excessos e a se distanciar de novas propostas de trabalho, evitando as patologias da sobrecarga de trabalho, o que conduz ao adoecimento (DEJOURS, 2007).

Mesmo com tantos desafios e com dúvidas referentes ao conceito e prática dessa metodologia, a equipe considerou possível realizar e incluir o AM na sua agenda. Mas fizeram uma ressalva: seria possível realizar o AM desde que se reconhecesse os limites de cada equipe e profissional, de forma que as ações pudessem acontecer respeitando a realidade da rede de saúde. Por isso responderam que era possível, mas deixaram em evidência a preocupação referente às dificuldades da equipe devido ao número reduzido de profissionais.

\section{Propostas para tornar viável o AM}

Quadro 5. IC* e DSC ${ }^{\star \star}$ sobre as propostas para viabilizar o $\mathrm{AM}^{\star \star \star}$

\begin{tabular}{ll}
\hline A equipe necessita de planejamento e mais profissionais para superar as dificuldades do AM \\
IC 14 \\
(DSC) - "Quando eu chego para fazer um matriciamento, vejo que é difícil deslocar a equipe, discutir em equipe, \\
entender a rede e a complexidade do caso. Por isso, acho que tem que ser algo bem organizado e com mais pro- \\
fissionais" (P2). \\
\hline \\
Formação de uma equipe para articular a rede de saúde \\
(DSC) - "Eu acho que deveria ter uma equipe que conhecesse todos os equipamentos de saúde que existem no \\
Município. Poderia ser uma equipe que realizasse um trabalho de um ano, só para fazer isso. Assim poderia forta- \\
lecer toda a rede, desenhar a rede e ajudar a organizar esse fluxo" (P8). \\
O AM necessita de uma equipe específica \\
IC $16 \quad$ (DSC) - "Eu vejo o grande problema que parte de cima, dos gestores. Porque teria que ter uma equipe que fosse \\
específica para realizar o apoio matricial" (P6).
\end{tabular}

${ }^{\star} \mid \mathrm{C}$ - Ideia Central / ${ }^{\star \star}$ DSC - Discurso do Sujeito Coletivo / ${ }^{* \star \star} \mathrm{AM}$ - Apoio Matricial.

Para realizar o matriciamento, seria necessário planejamento e organização. Para executar uma metodologia com mais de uma equipe, o envolvimento de profissionais foi avaliado como fundamental. Essa responsabilidade não poderia ser voltada apenas 
para o gestor, sustentando um lugar subjetivo exclusivo da gestão para determinar as ações, estratégias e intervenções a serem realizadas. Em relação a esse desafio, Campos (1998) sugere a cogestão, um método de gestão que permita e estimule os trabalhadores a ampliar sua capacidade de reflexão e autonomia, de forma a que todos participem das decisões governando juntos, com uma gestão participativa. Deve ser uma gestão que estimule a produção da liberdade e incentive as pessoas a assumirem riscos, promovendo o prazer da criação e motivando os envolvidos a estar preparados para o compromisso.

Assim, o AM é uma metodologia de trabalho que necessita da participação de todos os atores envolvidos na produção de saúde, como gestores, profissionais de saúde, usuários, familiares e comunidade, que pactuam as ações de saúde a partir de um planejamento, de forma a compartilhar as responsabilidades no processo de reabilitação psicossocial e nos espaços sociais (MIELKE; OLCHOWSKY, 2010).

Por fim, foi construída a proposta de se criar uma equipe específica para realizar o AM, embora deva ser discutida com cautela. Um dos principais eixos fundamentais do modelo médico hegemônico é a fragmentação do cuidado por especialidade. Assim, a solicitação de uma equipe especializada em AM reflete a mesma lógica do modelo hegemônico, mas, dessa vez, relacionada à organização do trabalho.

Em outras pesquisas (ELIZAETE, 2009; GARCIA; ÁVILA-NASCIMENTO，2012; PRESTES，2011; FIGUEIREDO, 2006), as equipes dos Caps utilizaram como metodologia de cuidado o AM. São exemplos que apresentam uma forma de organização, assim como o Nasf, que, entre outras metodologias, também utiliza a metodologia do AM (BRASIL, 2011).

Portanto, como já analisado, o matriciamento é uma metodologia que visa ao compartilhamento de conhecimentos, técnicas e experiências, não se limitando a funções de uma equipe específica. Para exemplificar empregando uma comparação, esperar que uma equipe específica tenha o uso exclusivo do AM seria o mesmo que imaginar que apenas a EqSF realizasse clínica ampliada, construísse Projeto Terapêutico Singular, fizesse vínculo com os usuários ou realizasse escuta qualificada. Assim, como tais, o AM é uma metodologia que deve ser utilizada por todas as equipes de saúde.

\section{Conclusão}

Esta pesquisa evidenciou que um conceito não compreendido em sua totalidade pelas equipes resulta na criação de obstáculos imaginários, impossibilitando o desenvolvimento de potentes ações de cuidado integral e continuado. Por não ter claro um conceito, a equipe que oferta o AM pode se sentir insegura e solicitar apoio e incentivos da gestão e de outras equipes.

Ainda, a equipe percebe um distanciamento entre teoria e prática, demonstrando dificuldade para a produção de liberdade e compromisso diante de situações que exigem a criação de um novo conhecimento ou técnica. Afinal, é preciso reinventar os cuidados de saúde, tendo em vista que a prática da clínica ampliada exige a adoção de um cuidado singular, contrário à padronização e repetição técnica.

Em relação às experiências com AM, a equipe escutou e observou as experiências de outras equipes de Capsi, possibilitando o início de uma construção teórica sobre o tema. Assim, foi possível concluir que a equipe não apresentou o conceito claro, mas teve uma primeira aproximação, demonstrando que a metodologia não era completamente desconhecida.

As dúvidas referentes à responsabilidade do AM aparecem na dificuldade de a equipe nomear, por variadas circunstâncias, quem seriam os protagonistas a utilizar o instrumento. Concluem que há a necessidade de implantar uma equipe específica para 
realizar um trabalho que fortaleça e articule a rede de saúde.

A equipe identificou a necessidade de incentivos para aprofundar tal conhecimento e experiência, o que instigou os pesquisadores a olharem para a organização e articulação de uma rede que realmente consiga contribuir com a mudança de paradigma da saúde mental infanto-juvenil.

Portanto, diante da mudança de paradigma da saúde mental, tem-se ainda um longo caminho a percorrer nesse trajeto da reforma, em particular, na área da infância e adolescência. Essa população precisa ganhar visibilidade social e no campo da saúde para que se efetive um cuidado em saúde como um direito e que se possam construir ações

\section{Referências}

ANDRADE, S. M.; DUARTE, S. J. H.; MAMEDE, M. V. O. Opções teórico metodológicas em pesquisas qualitativas: representações sociais e discurso do sujeito coletivo. Saúde Soc., São Paulo, v. 18, n. 4, p. 620626, 2009.

ARONA, E. C. Implantação do Matriciamento nos Serviços de Saúde de Capivari (SP). Saúde Soc., São Paulo, v. 18, supl. 1, 2009.

BACKES, D. S. et al. Grupo focal como técnica de coleta e análise de dados em pesquisas qualitativas. São Paulo: Mundo Saúde, v. 35, n. 4, p. 438-442, out./dez. 2011.

BOWLBY, J. Cuidados maternos e saúde mental. São Paulo: Martins Fontes, 2002. visando à integralidade, à singularidade e à subjetividade das crianças e dos adolescentes. Espera-se, então, que este artigo colabore com tal proposta e contribua para o crescimento e desenvolvimento da equipe participante desta pesquisa e de outras equipes de saúde e para tantos outros estudos na área.

\section{Colaboradores}

Ambos autores participaram de todas as etapas do processo. Assim, contribuíram para a concepção, planejamento e análise dos dados; elaboração do rascunho e revisão crítica do conteúdo; bem como aprovação da revisão final do manuscrito.
BRASIL. Ministério da Saúde. Portaria nº 2488, de 21 de outubro de 2011. Política Nacional de Atenção Básica, estabelecendo a revisão de diretrizes e normas para a organização da Atenção Básica, para a Estratégia Saúde da Família (ESF) e o Programa de Agentes Comunitários de Saúde (PACS). Diário Oficial [da] União, Brasília, DF, 2011. Disponível em: <http:// www.saude.mt.gov.br/atencao-a-saude/arquivo/2581/ portarias>. Acesso em: 31 out. 2016.

. Ministério da Saúde. Secretaria de Atenção à

Saúde. Caminhos para uma política de saúde mental infanto-juvenil. Brasília, DF: Ministério da Saúde, 2005.

CAMPOS, G. W. S. O anti-Taylor: sobre a invenção de um método para co-governar instituições de saúde produzindo liberdade e compromisso. Cad. Saúde Publ., 
Rio de Janeiro, v. 14, n. 4, p. 863-870, 1998.

Equipes de referência e apoio especializado matricial: um ensaio sobre a reorganização do trabalho em saúde. Ciênc. Saúde Colet., Rio de Janeiro, v. 4, n. 2, p. 393-403, 1999.

CAMPOS, G. W. S.; DOMITTI, A. C. Apoio matricial e equipe de referência: uma metodologia para gestão do trabalho interdisciplinar em saúde. Cad. Saúde Públ., Rio de Janeiro, v. 23, n. 2, fev. 2007.

CAVALCANTE, C. M.; JORGE, M. S. B.; MOURASANTOS, D. C. Onde está a criança? Desafios e obstáculos ao apoio matricial de crianças com problemas de saúde mental. Physis, Rio de Janeiro, v. 22, n. 1, p. 161-178, 2012

\section{CECÍLIO, L. C. O.; MATSUMOTO, N. F. Uma}

taxonomia operacional de necessidades de Saúde. In: PINHEIRO, R.; FERLA, A. F.; MATTOS, R. A. (Org.). Gestão em Redes: tecendo os fios da integralidade em saúde. Rio Grande do Sul: Educs; UFRS; IMS; Uerj; Cepesc, 2006. p. 37-50.

CHIAVERINI, D. H. et al. Guia prático de matriciamento em saúde mental. Brasília, DF: Ministério da Saúde, 2011.

DEJOURS, C. A psicodinâmica do trabalho na pósmodernidade. In: MENDES, A. M.; LIMA, S. C. C.; FACAS, E. P. Diálogos em psicodinâmica do trabalho. Brasília, DF: Paralelo, 2007. p. 13-26.

FIGUEIREDO, M. D. Saúde Mental na Atenção Básica: um estudo hermenêutico-narrativo sobre o Apoio Matricial na rede SUS. 2006. Dissertação (Mestrado) - Programa de Pós-Graduação em Saúde Coletiva, Unicamp, Campinas, 2006.
GARCIA JÚNIOR, C. A. S.; ÁVILA-NASCIMENTO, P. T. O dispositivo apoio matricial na atenção primária em saúde: um relato de experiência no município de João Pessoa/PB. Rev. Saúde Públ., Florianópolis, v. 5, n. 2, p. 93-104, maio/ago. 2012.

LEFÉVRE, F.; LEFÉVRE, A. M. C.; MARQUES, M. C. C. Discurso do sujeito coletivo, complexidade e organização. Ciênc. Saúde Colet., São Paulo, v. 14, n. 4, p. 1193-1204, 2009

MERHY, E. E. Engravidando palavras: o caso da integralidade. In: FRANCO, T. B.; MERHY, E. E. Trabalho, produção do cuidado e subjetividade em saúde. São Paulo: Hucitec, 2013. p. 252-265.

MIELKE, F. B.; OLCHOWSKY, A. Saúde mental na Estratégia da Família: avaliação de apoio matricial. Versão Brasileira de Enfermagem, Brasília, DF, v. 63, n. 6, p. 900-907, nov./dez. 2010.

PRESTES, L. I. N. Apoio Matricial: um Caminho de Fortalecimento das Redes de Atenção à Saúde em Palmas (TO). Revista Brasileira de Ciências da Saúde, João Pessoa, v. 15, n. 2, p. 215-218, 2011.

RANÑA, W. A saúde mental da criança na atenção básica: detecção e intervenção a partir do Programa de saúde da Família e do apoio matricial. In:

LAURIDSEN-RIBEIRO, E.; TANAKA, O. Y. Atenção em saúde mental para crianças e adolescentes no SUS. São Paulo: Hucitec, 2010. p. 170-185.

Recebido para publicação em abril de 2016

Versão final em setembro de 2016

Conflito de interesses: inexistente

Suporte financeiro: não houve 